\title{
Enhancement of Social based Routing Protocol in Delay Tolerant Networks
}

\author{
Chirag M. Patel \\ Department of Information Technology \\ G H Patel College of Engineering and Technology, \\ Vallabh Vidyanagar, Gujarat, India
}

\author{
Nikhil Gondaliya \\ Department of Information Technology \\ G H Patel College of Engineering and Technology, \\ Vallabh Vidyanagar, Gujarat, India
}

\begin{abstract}
Delay Tolerant Network has lack of continuous network connectivity, so probability of end to end path between source and destination for any given time is low. Routing in DTN is challenging because it consists a network partitioning, long delay and unstable topology. DTN use store-carry-forward approach for routing, in that packet stored until it encountered relay node to forward. In recent years, social based approaches have drawn much interests in routing design which exploits a social behavior of the node to make better routing decision. Social Network Analysis mainly focuses on studying the relationship among social entities, patterns and implication of their relationship. By using social properties, we can improve routing performance. SimBet is social based routing protocol, which use two social properties named Similarity and Betweenness Centrality for routing packets. We have proposed SimBet multi-copy routing scheme that transferred a number of copies of a message during contact opportunity is proportional to the ratio of SimBetUtility values of nodes. We have selected ONE Simulator for examining the results of different DTN routing protocols. We present a simulation using real trace data like Cambridge and pmtr. From the results of simulation, we conclude that our applied SimBet multi-copy performs better than SimBet and epidemic in terms of delivery ratio, it has lower latency and overhead ratio than SimBet and epidemic for larger buffer size and TTL, where still its hop count is higher than SimBet.
\end{abstract}

\section{General Terms}

Routing scheme, Performance.

\section{Keywords}

Delay Tolerant Network, Social Based Routing, Multi-copy, SimBet.

\section{INTRODUCTION}

Delay Tolerant Network is also referred as the Intermittently Connected Mobile Network. DTN nodes are mobile nodes, the connectivity of the network is maintained by nodes when they comes in the transmission ranges of other nodes. If any node has message to send and it is not connected with other nodes, it stores the message in buffer space until it comes in contact with another node. The primary motivation behind the DTN is to route packet for message delivery in such challenged Networks like those operated in mobile environment, extreme terrestrial environment and planned network in space. Routing challenges in DTN are bandwidth, mobility, buffer space, security and energy. DTN has characteristics like Intermittent Connectivity, Long or Variable Delay, Asymmetric Data Rates and High error rates. Due to these characteristic, classical ad hoc routing protocol not being applicable for DTN [1]. The DTN routing protocols are categorized as: 1. Flooding based, 2. Forwarding based and 3. Social based. In flooding based, copies of messages are flooded into network without knowledge of network. In forwarding based, knowledge about the network is used to calculate a best path of nodes between source and destination. In social based, it exploit social behaviors of DTN nodes to make better routing decision. For better routing performance, we can consider social properties like Community, Centrality, Similarity, and Friendship as positive social characteristics which benefit the relay selection and negative social characteristics like Selfishness which degrade the performance of network. Mobile nodes are used and carried by people in most of DTN application, whose behavior are describe by social models. Social Network Analysis (SNA) mainly focuses on studying relationship among social entities and patterns and implication of their relationships [2]. SimBet is social based routing protocol, which use the concept of ego networks where nodes are not required to exchange information about the entire network topology, but only locally available information is considered [6]. Encounter Based Routing (EBR) [5] protocol sends a number of copies of messages during a contact opportunity is proportional to the ratio of the Encounter Values of the nodes. By utilizing a concept of EBR, we have proposed SimBet multi-copy routing scheme that sends a number of copies of messages based on proportion of SimBetUtility values of the nodes.

The rest of paper is organized as follows: Section 2 give reviews about related work in area of routing protocols in DTNs. Section 3 introduces the proposed routing scheme SimBet multi-copy with pseudo code. Section 4 shows the simulations and results of applied SimBet multi-copy, SimBet and Epidemic on data set Cambridge and pmr in terms of delivery ratio, overhead ratio, latency and hop count.

\section{RELEATED WORK}

Epidemic [3] routing simply flooding the messages into network. This approach does not require information about the network, but it requires large amount of buffer size, bandwidth and power. It has the highest delivery ratio with unlimited buffer space and high overhead due to flooding of messages. Spray and Wait [4] routing sprays a number of copies into the network and afterward waits till one of these nodes meets the destination. Binary spray and wait [4] initially starts with some number of copies and any node that has more than one copy of message and encounters another node with no copies, then it hands over to another node half copies and keeps half copies itself, when it is left with only one copy, then it switches to direct transmission. Encounter based routing (EBR) [5] route decisions on the base of encounter rate of nodes, inclination to message transferred with nodes that have higher encounter rates. Encounter Value (EV) represents the node's past rate of encounters as an 
exponentially weighted moving average. The number of copies of a message transferred during a contact opportunity is proportional to the ratio of the EVs of the nodes. Our proposed routing scheme is based upon previous work SimBet [6] which is a social routing protocol, that sends a packet based pre-estimated betweenness centrality metrics and locally determined social similarity to destination node. SimBetAge [7] exploit the lightweight and ego centric scheme of SimBet and consider strength and gradual aging of social relations into account. Bubble Rap [8] focus on two key social metrics: community and centrality for routing decision. [9] Introduce that efficient routing scheme by using a node's local contact history and social network metrics. Social-Similaritybased Routing (SOSIM) [10] uses similarity metrics based on nodes' contact history to evaluate more accurately the social similarity between nodes and guide forwarding towards the destination. In Friendship Based [11] Routing scheme, there is metric for detecting a quality of friendships. Using this metric each node defines its friendship community. Friendship metric is used to make the better forwarding decisions.

\subsection{Background on SimBet Routing [6]}

SimBet routing is based on two social properties named betweenness centrality and similarity. In SimBet routing algorithm, forwarding decisions are based on local calculation and it makes no assumption of global knowledge. "Similarity is measure as number of common neighbor between current node and destination node". Centrality can be measure in 3 ways: 1. "Degree Centrality is a measure of direct ties that involve a given node". 2. "Closeness Centrality is measure as reciprocal of mean geodesic distance, which is shortest path between a current node and all other reachable nodes". 3 . "Betweenness Centrality is measure the extent to which a node lies on the paths linking other nodes". Betweenness centrality can be considered as a measure of the extent to which a node has control over information flowing between others. A node with a high betweenness centrality has a more capacity to facilitate interactions between the nodes that it links. "Betweenness centrality is calculated using an ego network representation of the nodes with which the ego node has come into contact". Betweenness centrality is calculated for given node $\mathrm{p}_{\mathrm{i}}$ is:

$$
C_{B}\left(p_{i}\right)=\sum_{j=1}^{N} \sum_{k=1}^{j-1} \frac{g_{j k}\left(p_{i}\right)}{g_{j k}}
$$

Where $\mathrm{g}_{j k}$ is the total number of geodesic paths linking $\mathrm{p}_{j}$ and $\mathrm{p}_{k}$, and $\mathrm{g}_{j k}\left(\mathrm{p}_{i}\right)$ is the number of those geodesic paths that include $\mathrm{p}_{\mathrm{i}}$. Similarity is a number of common neighbours between current node and destination node. Similarity of $u$ to $\mathrm{v}$ can be defined as the number of common neighbour between $\mathrm{u}$ and $\mathrm{v}$ :

$$
\mathrm{S}_{\mathrm{u}}(\mathrm{v})=|\mathrm{N} 1(\mathrm{u}) \cap \mathrm{N} 1(\mathrm{v})|
$$

The similarity utility and betweenness utility of node $\mathrm{n}$ for delivering message to destination node $\mathrm{d}$ compared to node $\mathrm{m}$ is given by:

$$
\begin{aligned}
\operatorname{SimUtil}_{\mathrm{n}}(\mathrm{d}) & =\frac{\operatorname{Sim}_{\mathrm{n}}(\mathrm{d})}{\operatorname{Sim}_{\mathrm{n}}(\mathrm{d})+\operatorname{Sim}_{\mathrm{m}}(\mathrm{d})} \\
\text { BetUtil }_{\mathrm{n}} & =\frac{\operatorname{Bet}_{\mathrm{n}}}{\operatorname{Bet}_{\mathrm{n}}+\operatorname{Bet}_{\mathrm{m}}}
\end{aligned}
$$

$$
\operatorname{SimBetUtil}_{n}(\mathrm{~d})=\alpha \operatorname{SimUtil}_{n}(\mathrm{~d})+\beta \text { BetUtil }_{n}
$$

Where $\alpha$ and $\beta$ are tunable parameter which adjust the relative importance of the two utility values. Node with higher SimBetUtilty value will get message to forward

\section{PROPOSED ROUTING SCHEME} 3.1 SimBet multi-copy routing protocol:

In SimBet routing scheme, packet is sent based on SimBetUtility, it means node with higher SimBetUtility will get packet to forward. But in our proposed scheme, a number of copies of a message transferred during a contact opportunity is proportional to the ratio of the SimBetUtility values of the nodes. For example, when two nodes $\mathrm{n}$ and $\mathrm{m}$ encountered, for every message $\mathrm{Mi}$, node $\mathrm{n}$ sends

$$
\mathrm{m}_{\mathrm{i}} \frac{\text { SimBetUtil }_{\mathrm{m}}}{\text { SimBetUtil }_{\mathrm{n}}+\text { SimBetUtil }_{\mathrm{m}}}
$$

Number of copies of message $\mathrm{Mi}$, where $\mathrm{mi}$ is the total number of Mi copies stored at node $\mathrm{n}$. and sender node $\mathrm{n}$ has following copies of message

$$
\mathrm{m}_{\mathrm{i}}-\mathrm{m}_{\mathrm{i}} \frac{\text { SimBetUtil }_{\mathrm{m}}}{\text { SimBetUtil }_{\mathrm{n}}+\text { SimBetUtil }_{\mathrm{m}}}
$$

For example assume node $\mathrm{n}$ has 6 copies of message Mi with SimBetUtiln $=0.25$, comes in contact with node $\mathrm{m}$ with SimBetUtilm $=0.65$. Now node $\mathrm{n}$ sends $60.65 /(0.65+0.25)=$ 4.33333 , which converted into 4 by Math.Foor (4.33333) to node $\mathrm{m}$. and node $\mathrm{n}$ has $6-4=2$ copies of messages.

\subsection{Pseudo code for node $n$}
1: When node $n$ and $m$ encounter do
2: for all messages $m_{i}$ in msgQueue of node $n$
3: if $\operatorname{msgQueue}\left(m_{i}\right) \cdot \operatorname{hasMsgForDest}(m)==$ true then
4: deliverMsgs $\left(m_{i}, m\right)$
5: end if
6: end for
7: requestEncounters $(m)$
8: done
9:

10: upon reception of encounter vector $e V$ from node $m$ do

11: addNodeEncounters $(m, e V)$

12: updateBetweenness()

13: updateSimilarity()

14: for all messages $m_{i}$ in msgQueue of node $n$

15: $d_{i}=\operatorname{msgDest}\left(m_{i}\right)$

16: $\quad n_{i}=\operatorname{noCopies}\left(m_{i}\right)$

17: $\quad \operatorname{nCopy}_{i}=n_{i}-n_{i} * \operatorname{SimBetUtil}_{m}\left(d_{i}\right) / \operatorname{SimBetUtil}_{n}\left(d_{i}\right)+$ $\operatorname{SimBetUtil}_{m}\left(d_{i}\right)$ 18: $\operatorname{mCopy}_{i}=n_{i} * \operatorname{SimBetUtil}_{m}\left(d_{i}\right) / \operatorname{SimBetUtil}_{n}\left(d_{i}\right)+$
$\operatorname{SimBetUtil}_{m}\left(d_{i}\right)$

19: node $\mathbf{n}$ sends $\mathbf{m C o p y}$ to node $\mathbf{m}$

20: $\quad \operatorname{sendMsgs.add}\left(m_{i}\right)$

21: end for

22: transferMsg(sendMsgs, $m)$ 


\section{3: done}

\section{SIMULATIONS}

In this section we describe the simulation used to evaluate epidemic, SimBet, and our applied SimBet multi-copy using real trace data cambridge and pmtr. We choose ONE (Opportunistic Network Environment) simulator [12] for evaluating this routing protocols.

\subsection{Performance Metrics}

1. Delivery ratio, which is defined as the ratio of number of messages successfully delivered $\left(\mathrm{M}_{\text {Delivered }}\right)$ to destination to the total number of messages generated $\left(\mathrm{M}_{\text {Total }}\right)$.

$$
\text { Delivery ratio }=\frac{\mathrm{M}_{\text {Delivered }}}{\mathrm{M}_{\text {Total }}}
$$

2. Overhead ratio, which is defined as the ratio of total number of relayed messages subtract delivered messages to the total number of messages delivered.

$$
\text { Overhead ratio }=\frac{\mathrm{M}_{\text {Relay }}-\mathrm{M}_{\text {Delivered }}}{\mathrm{M}_{\text {Delivered }}}
$$

3. Average latency, which is defined as the average delay needed to deliver a message to the destination.

4. Average hop count, which is defined as the average number of hops executed by the message to reach the destination from the source.

We consider performance metrics such as delivery ratio, overhead ratio, average latency, and average hop count. For better performance on successful message delivery, delivery ratio should be higher.

High overhead in network is due to additional flooded message. Therefore, both delivery rate and overhead are analyzed simultaneously. Latency is the time required by message from send source to destination. Finally, average number of hop count is compared to demonstrate consumption of resources. DTNs accept a tolerable latency for delivery of message to destination. But some applications do not agree with the extremely higher number of hop count.

\subsection{Experimental Data Sets}

In this paper, for simulation we choose two experimental data sets Cambridge and pmtr that are taken from crawdad dataset as follow:

Cambridge: This data set includes a data of number of traces of Bluetooth sightings by groups of users carrying small devices (iMotes) for a number of days in campus environments. That small device iMotes were distributed mainly to two groups of students from the University of Cambridge Computer Laboratory that includes undergraduate first year and second year students, and also some $\mathrm{PhD}$ and postgraduate students. This experiment lasted for 11 days [13].

Pmtr: This dataset contains mobility traces from mobile devices at University of Milano. For this experiment, 49 Pocket Mobile Trace Recorders (PMTRs) were distributed to faculty members, $\mathrm{PhD}$ students, and technical staff. This experiment covers 19 days to be completed in November 2008 [14].

\subsection{Simulation Parameters}

In our simulation (one 1.5.1 simulator), we have assigned simple broadcast type as blue tooth interface with the transmit speed of $2 \mathrm{Mbps}$ for all the nodes. For making our simulation scenario comparable to constant application, we have used real trace data of Cambridge and pmtr for analyze the results of simulation. We have select value of $\alpha$ and $\beta$ is 0.5 for the simulation because we want to give equal importance to similarity and betweenness centrality.

As ONE simulator supports external event generator, we have set message event generator in such a way that it generates one new message in every 30 to 40 seconds. To evaluate the performance of the epidemic, SimBet and applied SimBet multi-copy routing, we have run the simulation for 987529 seconds for Cambridge and 1632979 seconds for pmtr for each routing protocol separately by varying buffer size from $1 \mathrm{MB}$ to $55 \mathrm{MB}$, message size from $8 \mathrm{~KB}$ to $1024 \mathrm{~KB}$ and TTL from $10 \mathrm{~m}$ to $1 \mathrm{w}$. Here TTL (Time to Live) values in, $\mathbf{m}$ for minutes, $\mathbf{h}$ for hours, $\mathbf{d}$ for days and $\mathbf{w}$ for weeks. The complete simulation setup information is given in Table 4.1.

Table 1: Parameters and their values utilized in Simulation for data set Cambridge and PMTR

\begin{tabular}{|c|c|c|}
\hline Experimental data set & Cambridge & pmtr \\
\hline Simulation Time & 987529 seconds & 1632979 seconds \\
\hline Interface & \multicolumn{2}{|c|}{ Bluetooth Interface } \\
\hline No of nodes & 36 & 49 \\
\hline Interface Type & \multicolumn{2}{|c|}{ Simple Broadcast } \\
\hline Transmit Speed & \multicolumn{2}{|c|}{$250 \mathrm{k}$ (2 Mbps) } \\
\hline Mobility & \multicolumn{2}{|c|}{ Real trace data } \\
\hline Buffer Size & $1,5,15,25,35,45,55$ in MB \\
\hline Routing Protocols & Epidemic, SimBet and SimBet_M \\
\hline Message Size & $8,16,32,64,128,256,512,1024$ in KB \\
\hline Event Interval & 30 to 40 seconds \\
\hline Message TTL & $10 \mathrm{~m}$ to $1 \mathrm{w}$ \\
\hline
\end{tabular}

\subsection{Result Analysis on data set Cambridge}

\subsubsection{Impact of varying buffer size:}

For varying buffer size, we have fixed the value of TTL to $2.5 \mathrm{~d}$ and message size to $128 \mathrm{~KB}$. As shown in the figure 1 , as buffer size increase, delivery ratio and average latency are increased because the node has more buffer space to store messages. As we have seen epidemic flooding the messages into the network, so overhead ratio is decreasing because of larger buffer space. Applied SimBet multi-copy has lower overhead ratio than SimBet after buffer size of $25 \mathrm{MB}$, so we conclude that for larger buffer size it has lower overhead ratio. Our applied SimBet multi-copy has higher delivery ratio and lower latency than others because of proper distribution of messages. From the results of simulation, we conclude that our applied SimBet multi-copy performs better than SimBet and epidemic in terms of delivery ratio, overhead ratio and average latency, but still its average hop count is higher than SimBet. 


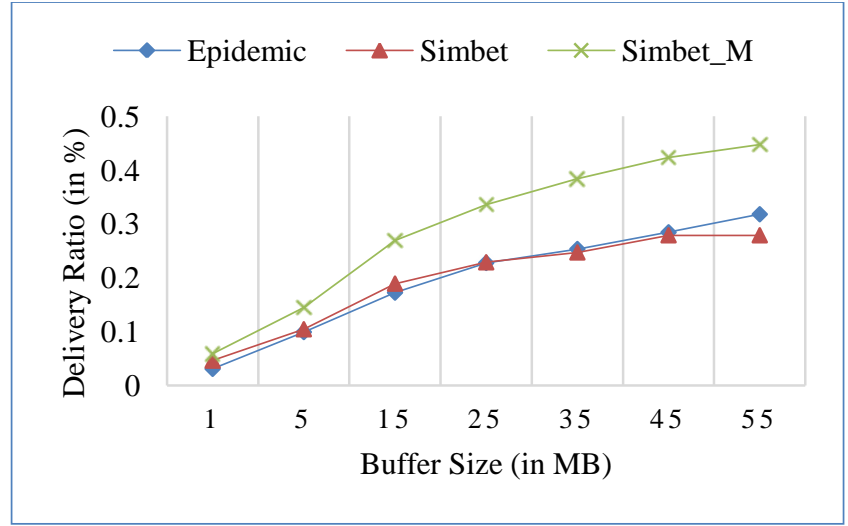

Figure 1(a)

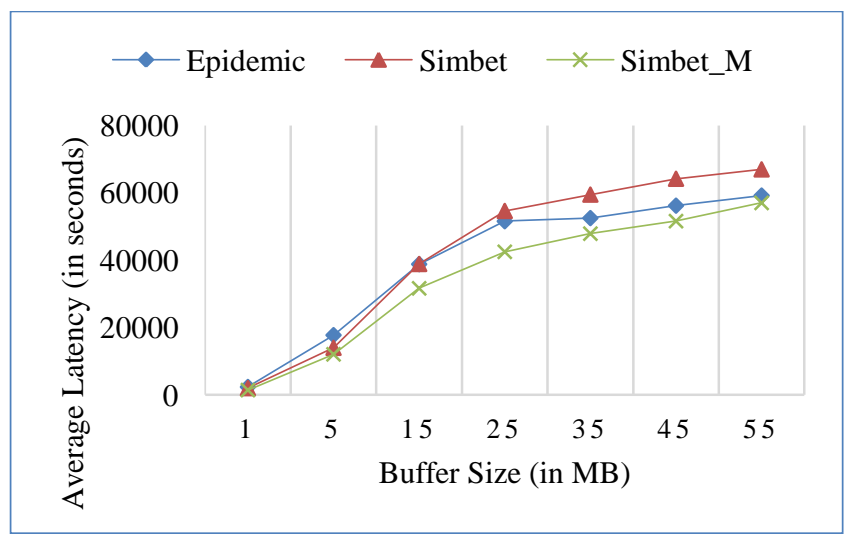

Figure 1(c)

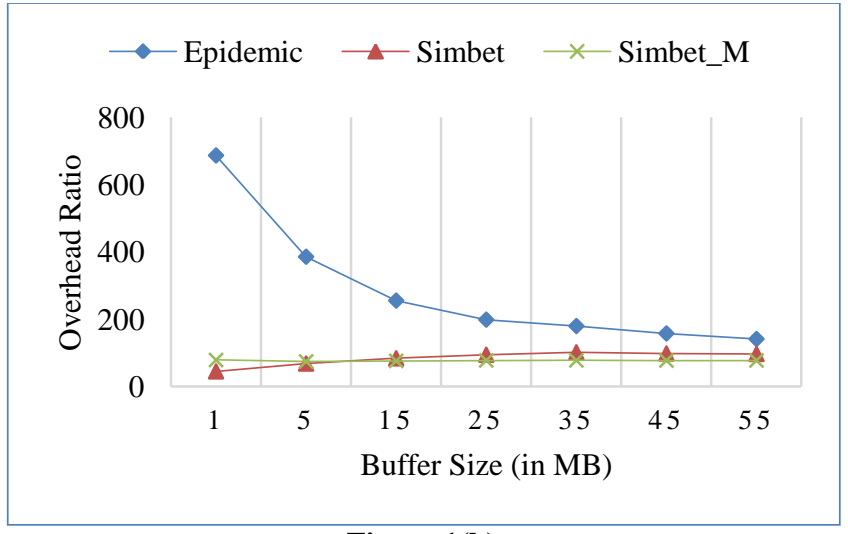

Figure 1(b)

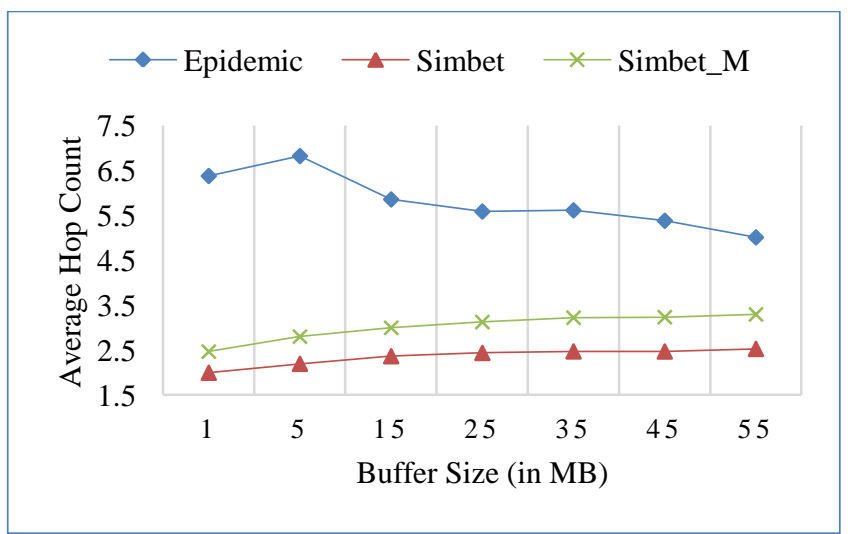

Figure 1(d)

Figure 1: Impact of varying buffer size on (a) Delivery Ratio (b) Overhead Ratio (c) Average Latency (d) Average Hop Count for Cambridge Data Set

\subsubsection{Impact of varying TTL}

For varying TTL, we have fixed the value of buffer size to $10 \mathrm{MB}$ and message size to $128 \mathrm{~KB}$. As shown in the figure 2, as TTL increase, delivery ratio, overhead ratio, average latency and average hop count are increased because message exists for longer time in the network. As we have seen epidemic flooding the message without any knowledge of network, therefore there is fluctuation in overhead ratio and

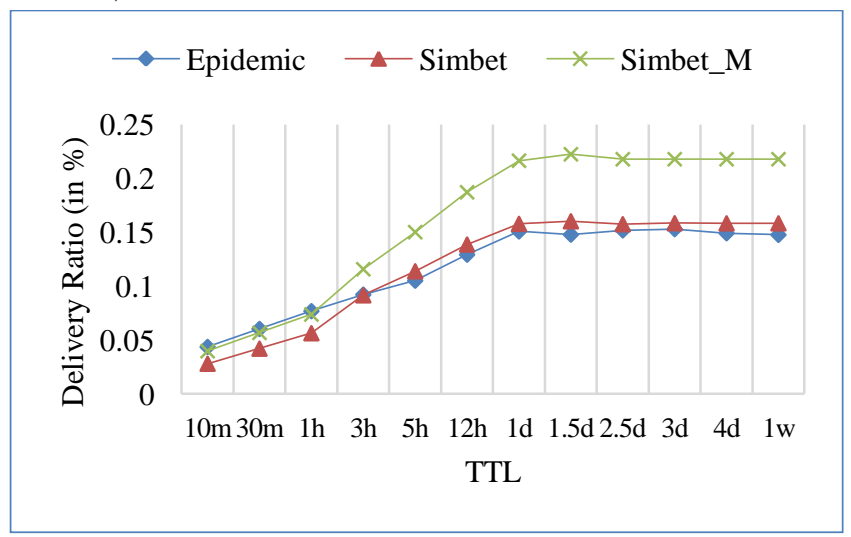

Figure 2(a) average hop count like from TTL 60 to 180 minutes. Our applied SimBet multi-copy has higher delivery ratio and lower average latency than others for larger TTL values. The overhead ratio of our applied SimBet multi-copy is lower than epidemic and similar to SimBet. So from the results of simulation, we conclude that our applied SimBet multi-copy performs better than SimBet and epidemic terms of delivery ratio and latency, but hop count is higher than SimBet.

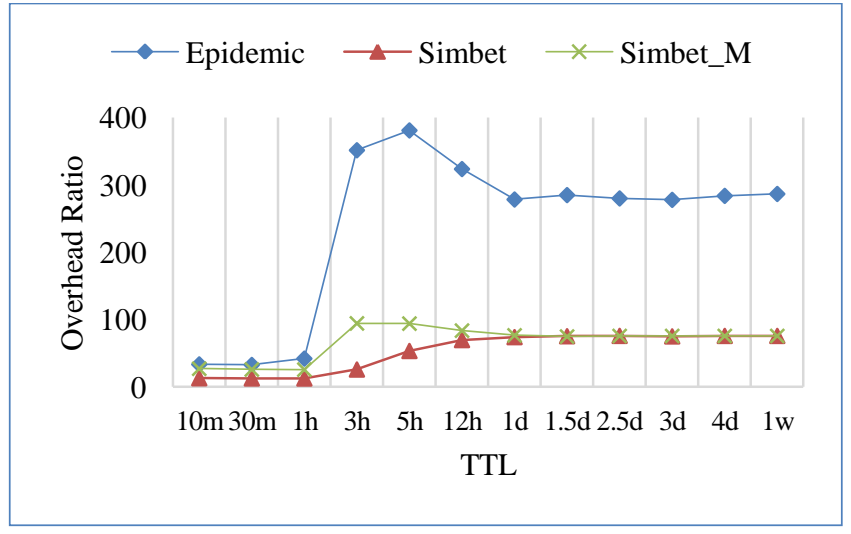

Figure 2(b) 


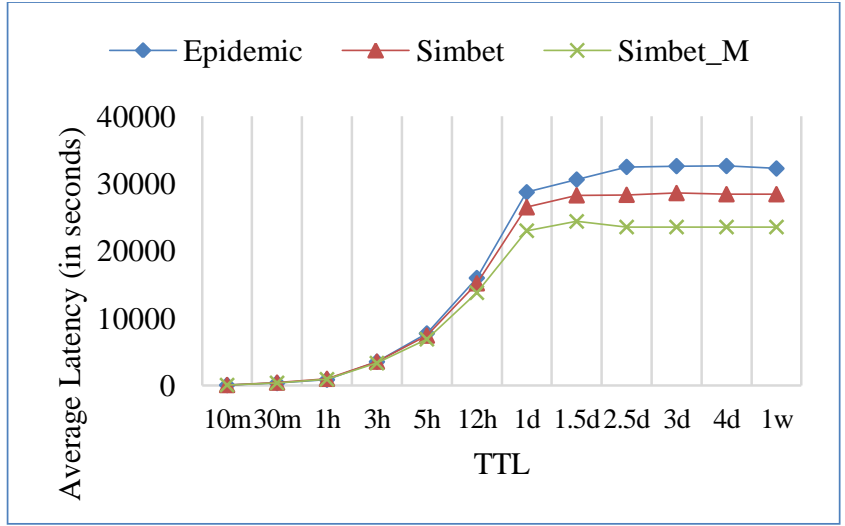

Figure 2(c)

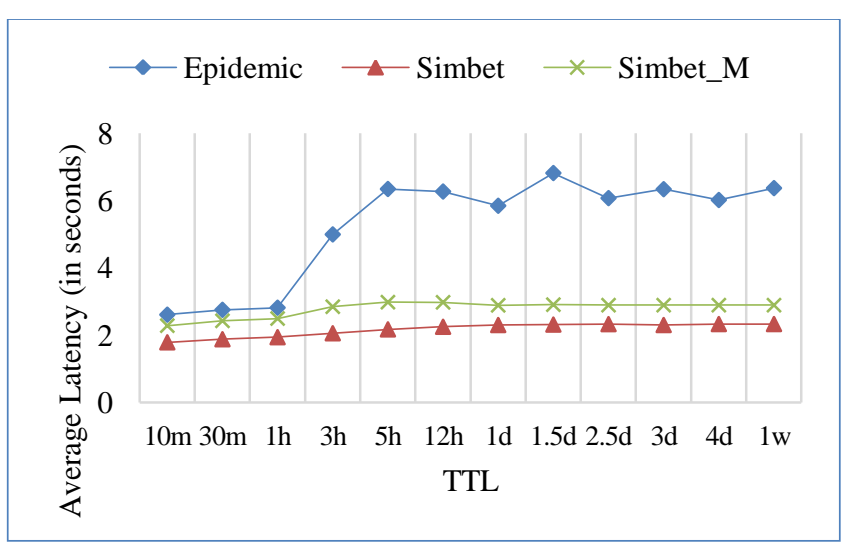

Figure 2(d)

Figure 2: Impact of varying TTL on (a) Delivery Ratio (b) Overhead Ratio (c) Average Latency (d) Average Hop Count for Cambridge Data Set

\subsection{Result Analysis on data set PMTR}

\subsubsection{Impact of varying buffer size:}

For varying buffer size, we have fixed the value of TTL to $5 \mathrm{~d}$ and message size to $128 \mathrm{~KB}$. As shown in the figure 3, as buffer size increase, delivery ratio and average latency are increased because the node has more buffer space to store messages. As we have seen epidemic flooding the messages into the network, so overhead ratio is decreasing because of larger buffer space. Applied SimBet multi-copy has lower

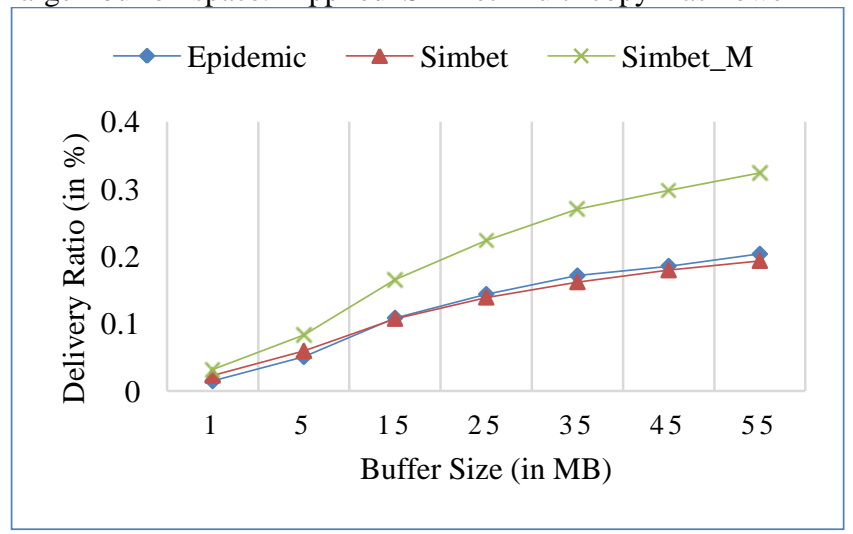

Figure 3(a)

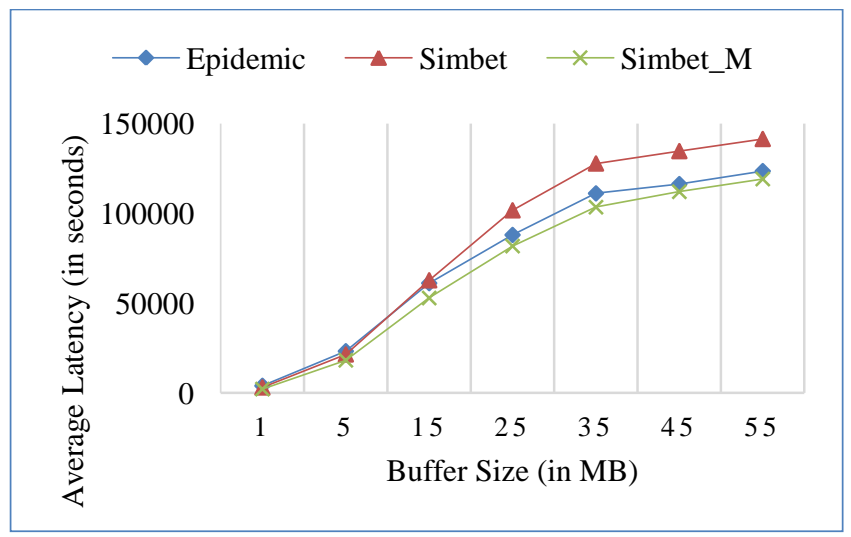

Figure 3(c) overhead ratio than SimBet after buffer size of $5 \mathrm{MB}$, so we conclude that for larger buffer size it has lower overhead ratio. Our applied SimBet multi-copy has higher delivery ratio and lower latency than others because of proper distribution of messages. From the results of simulation, we conclude that our applied SimBet multi-copy performs better than SimBet and epidemic in terms of delivery ratio, overhead ratio and average latency, but still its average hop count is higher than SimBet.

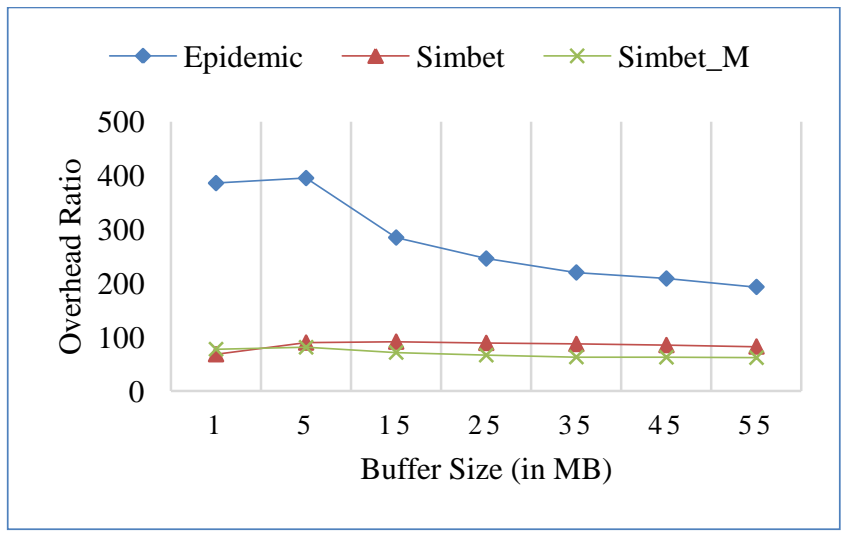

Figure 3(b)

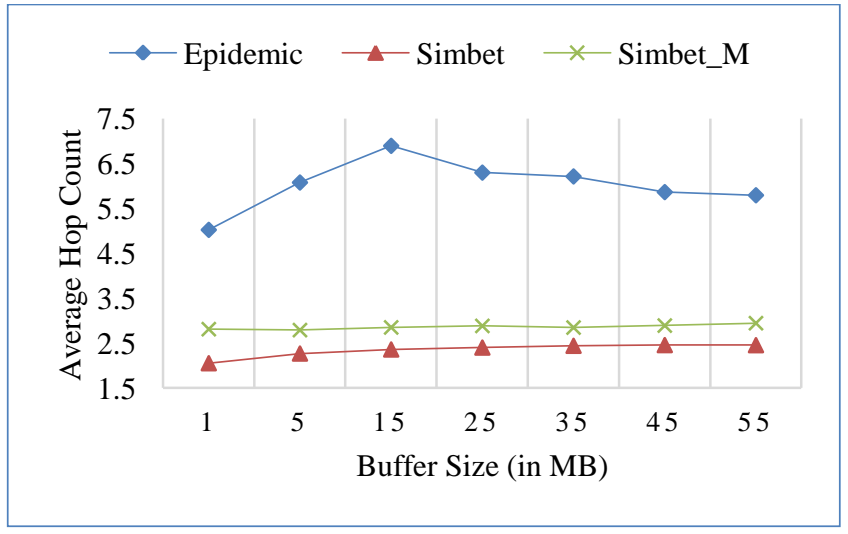

Figure 3(d)

Figure 3: Impact of varying Buffer Size on (a) Delivery Ratio (b) Overhead Ratio (c) Average Latency (d) Average Hop Count for pmtr Data Set 


\subsubsection{Impact of varying TTL:}

For varying TTL, we have fixed the value of buffer size to $10 \mathrm{MB}$ and message size to $128 \mathrm{~KB}$. As shown in the figure 4, as TTL increase, delivery ratio, overhead ratio, average latency and average hop count are increased because message exists for longer time in the network. As we have seen epidemic flooding the message without any knowledge of network, therefore there is fluctuation in overhead ratio and average hop count like from TTL 60 to 180 minutes. Our

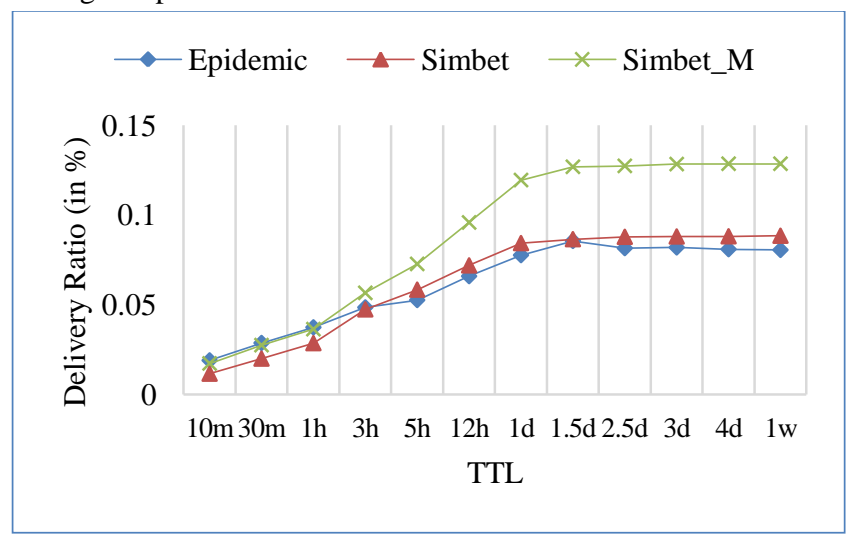

Figure 4(a)

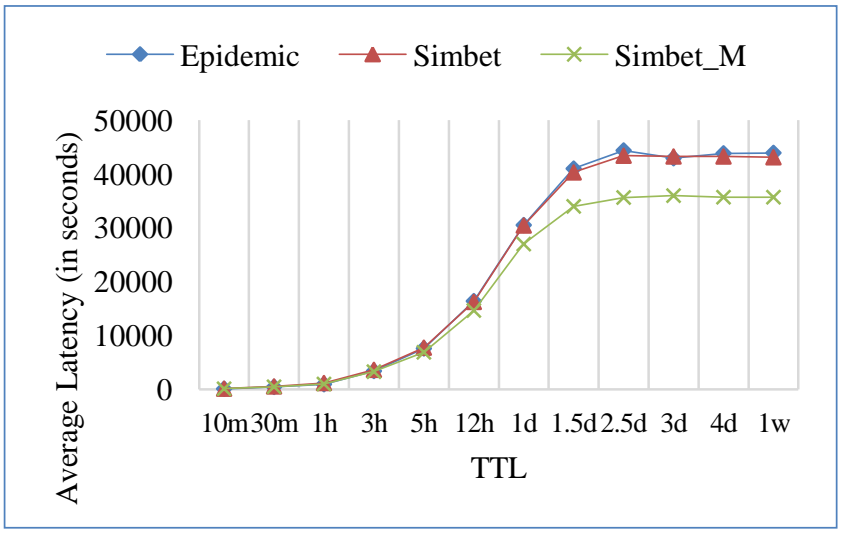

Figure 4(c) applied SimBet multi-copy has higher delivery ratio and lower average latency than others for larger TTL values. The overhead ratio of our applied SimBet multi-copy is lower than epidemic for all TTL values and lower than SimBet for larger TTL values. From the results of simulation, we conclude that our applied SimBet multi-copy performs better than SimBet and epidemic terms of delivery ratio, overhead ratio and latency for larger TTL values, and its hop count is higher than SimBet.

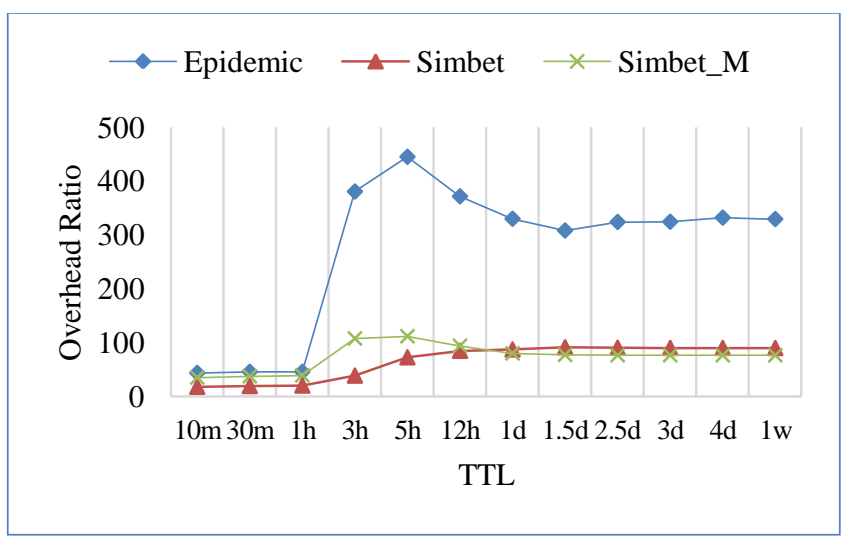

Figure 4(b)

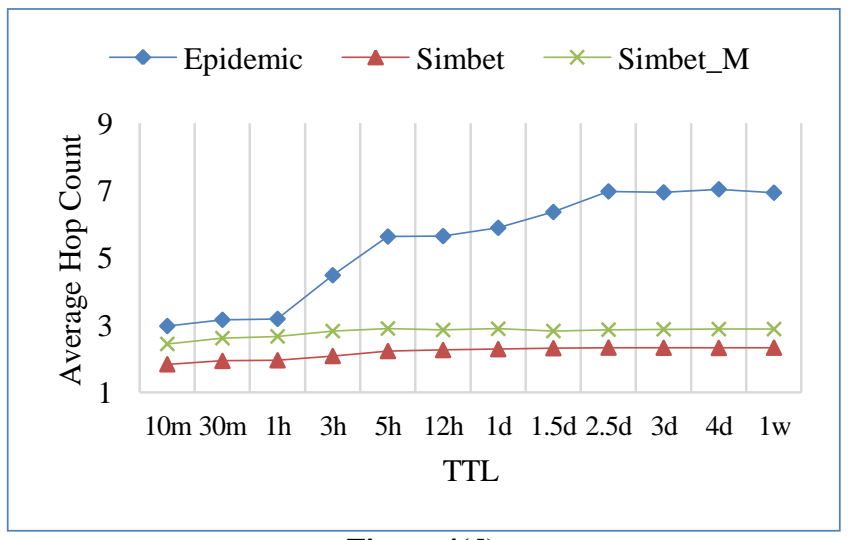

Figure 4(d)

Figure 4: Impact of varying TTL on (a) Delivery Ratio (b) Overhead Ratio (c) Average Latency (d) Average Hop Count for pmtr Data Set

\section{CONCLUSION AND FUTURE WORK}

DTN use flooding and forwarding scheme for routing messages. But from the analysis of the social pattern of mobile nodes which are carried by people, it found that social approaches gives a better routing performance than others. In this paper, we have introduced SimBet multi-copy routing scheme that sends a number of copies of a messages during encounter opportunity based on proportion of SimBetUtility values of the nodes by utilizing the concept of EBR. From the results of simulation, we conclude that our applied SimBet multi-copy performs better than SimBet and Epidemic in terms of delivery ratio, and also it has lower overhead and latency than SimBet and Epidemic for larger buffer space and TTL, but still its count is higher than SimBet. For future work, the overhead ratio of applied SimBet multi-copy can be decreased by applying buffer management scheme on it.

\section{ACKNOWLEDGMENTS}

We are extremely thankful to entire Information Technology Department, faculty and staff, for helping us in every conceivable way during my course of study for this work.

\section{REFERENCES}

[1] Suvarna Patil and Geetha R. Chillerge "Delay Tolerant Networks - Survey Paper International Journal of Engineering Research and Applications, ISSN : 22489622, Vol. 4, Issue 2( Version 2), February 2014.

[2] Zhu, Ying, et al. "A survey of social-based routing in delay tolerant networks: positive and negative social effects." Communications Surveys \& Tutorials, IEEE 15.1 (2013): 387-401.

[3] Vahdat, Amin, and David Becker. Epidemic routing for partially connected ad hoc networks. Technical Report CS-200006, Duke University, 2000.

[4] Spyropoulos, Thrasyvoulos, Konstantinos Psounis, and Cauligi S. Raghavendra. "Spray and wait: an efficient routing scheme for intermittently connected mobile networks." Proceedings of the 2005 ACM SIGCOMM workshop on Delay-tolerant networking. ACM, 2005. 
[5] Zhang, Junbao, et al. "Encounter-based routing in delay tolerant networks."Computational Problem-Solving (ICCP), 2011 International Conference on. IEEE, 2011.

[6] Daly, Elizabeth M., and Mads Haahr. "Social network analysis for routing in disconnected delay-tolerant manets." Proceedings of the 8th ACM international symposium on Mobile ad hoc networking and computing. ACM, 2007.

[7] Bitsch Link, Jó Ágila, et al. "SimBetAge: utilizing temporal changes in social networks for pocket switched networks." Proceedings of the 1st ACM workshop on User-provided networking: challenges and opportunities. ACM, 2009.

[8] Hui, Pan, Jon Crowcroft, and Eiko Yoneki. "Bubble rap: Social-based forwarding in delay-tolerant networks." Mobile Computing, IEEE Transactions on 10.11 (2011): 1576-1589.
[9] Kim, Chan-Myung, et al. "A Socially Aware Routing Based on Local Contact Information in Delay-Tolerant Networks." The Scientific World Journal 2014 (2014).

[10] Rothfus, Daniel, Christina Dunning, and Xiao Chen. "Social-similarity-based routing algorithm in Delay Tolerant Networks." Communications (ICC), 2013 IEEE International Conference on. IEEE, 2013.

[11] Eyuphan Bulut and Boleslaw K. Szymanski "Friendship Based Routing in Delay Tolerant Mobile Social Networks", Global Telecommunications Conference, 2010 IEEE

[12] Keranen, Ari. "Opportunistic network environment simulator." Special Assignment report, Helsinki University of Technology, Department of Communications and Networking (2008).

[13] http://crawdad.org/cambridge/haggle/

[14] http://crawdad.org/unimi/pmtr/ 